\title{
High-quality slitless radial velocities of extragalactic PNs with Subaru and FOCAS
}

\author{
R. H. Méndez, A. M. Teodorescu, and R.-P. Kudritzki \\ Institute for Astronomy, Univ. of Hawaii, 2680 Woodlawn Drive, Honolulu, HI 96822, USA \\ email: mendez@ifa.hawaii.edu
}

\begin{abstract}
We describe the implementation and accuracy of slitless radial velocity measurements of extragalactic PNs with Subaru and the FOCAS spectrograph. As a first application of the method, we have extended a previous study of PNs in NGC 4697 to larger angular distances from its center, failing again to find evidence of dark matter in this elliptical galaxy.
\end{abstract}

Keywords. planetary nebulae: general; techniques: radial velocities; galaxies: elliptical and lenticular, cD; galaxies: kinematics and dynamics

\section{Introduction}

Planetary nebulae (PNs) are excellent kinematic probes for dark matter and angular momentum distribution, particularly at large angular distances from the galaxy's center; see e.g. Romanowsky's review in these proceedings. But we need to measure the radial velocities of many PNs, and the shape of the PN luminosity function tells us that there is only a small number of bright PNs in any galaxy; therefore we need big telescopes to find the faint PNs. The most efficient instrument for slitless radial velocities is the Planetary Nebula Spectrograph (PN.S, Douglas et al. 2002). However, it has been designed for, and used at, the William Herschel $4.2 \mathrm{~m}$ telescope (on the Roque de los Muchachos, La Palma, Canary Islands, Spain); for that reason we have explored possible alternatives for slitless radial velocity work using $8 \mathrm{~m}$ telescopes. Our initial effort was based on the FORS spectrograph at the ESO VLT, Cerro Paranal, Chile; see Méndez et al. (2001), and Teodorescu et al. (2005). Later on, we have successfully tested a similar slitless radial velocity technique using the Faint Object Camera and Spectrograph (FOCAS) at the Cassegrain focus of the $8.2 \mathrm{~m}$ Subaru telescope on Mauna Kea, Hawaii, and here we would like to report our first FOCAS results, which involve an extension of the search for PNs in NGC 4697 up to a distance of 4 effective radii from its center.

\section{Slitless Spectroscopy with Subaru and FOCAS}

Planetary nebulae can be detected in the light of [O III] $\lambda 5007$ using the traditional on-band, off-band filter technique. Having taken the on-band image, insertion of a grism as dispersing element produces not only dispersion but also a displacement of all images. Calibration of this displacement as a function of wavelength and position in the CCD detector offers an efficient way of measuring radial velocities for all emission-line objects in the field, irrespective of their number and distribution. In the specific case of Subaru and FOCAS we use an echelle grism as dispersing element. With 175 grooves $/ \mathrm{mm}$, operating in the 4 th order, it gives a dispersion of $0.5 \AA$ /pixel, with good efficiency. Typical images with seeing around 0.5 arc second have a size of 5 pixels; this translates into a resolution of $140 \mathrm{~km} \mathrm{~s}^{-1}$, i.e. the PN's velocity field is not resolved. Assuming position errors of 0.2 pixel, the expected uncertainty in radial velocity is $5 \mathrm{~km} \mathrm{~s}^{-1}$. 

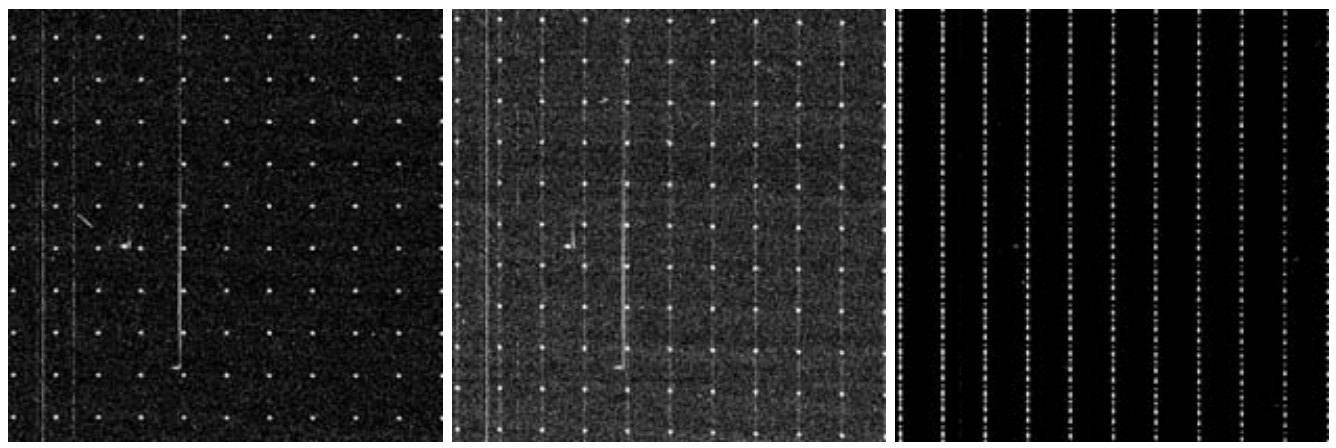

Figure 1. Calibration images obtained with FOCAS. From left to right, we show (a) the engineering mask illuminated by NGC 7293 through the on-band filter; (b) the same, adding the grism; what we see is [O III] $\lambda 5007$ at each position (the only emission line passed by the on-band filter), plus a very faint nebular continuum; and (c) the result of illuminating the mask with the Th-Ar comparison lamp. The direction of dispersion is vertical.

\section{Observations and calibrations}

The observations of the flattened elliptical galaxy NGC 4697 were made with FOCAS and the Subaru telescope on five nights, 2004 May 13/14 and 2005 May 12/13/14. The 2004 nights were partly unsuccessful due to bad weather. The field of view of FOCAS is $6^{\prime}$ and is covered by two CCDs of $2 \mathrm{k} \times 4 \mathrm{k}$ (pixel size $15 \mu \mathrm{m}$ ) with an unexposed gap of 5 arcsec between them. The image scale is $0.1 \operatorname{arcsec}_{\text {pixel }}{ }^{-1}$. Two FOCAS fields were observed, one on each side of NGC 4697 along its major axis, only partly overlapping the central zone, which had been previously observed with FORS (Méndez et al. 2001). Our purpose was to detect PNs in the remote outskirts of this galaxy, extending the earlier search to larger angular distances from its center. Full details of these observations, and a catalogue of the detected sources, will be published elsewhere.

In Fig. 1 we show an example of the calibration images. The first image is part of an engineering mask that produces an array of point sources when illuminated with the internal FOCAS lamps or any extended astronomical source. For our purposes we selected NGC 7293, whose large angular size guarantees illumination over the whole FOCAS field. The full mask provides almost 1000 calibration points, separated by about 100 pixels. The image on the left is NGC 7293 observed through the $\lambda 5007$ on-band filter and the engineering mask. The central image shows the effect of inserting the grism; comparing to the position of a fixed CCD defect, easily recognizable, it is clear that all the point sources have been displaced upwards. The last image shows what happens if we illuminate the mask with the Thorium-Argon comparison lamp; now each point source gives a Th-Ar spectrum. The positions of the Th and Ar emission lines permit to obtain the wavelength calibration.

This kind of observation permits to measure the radial velocities in NGC 7293 in two ways: (a) classical, using each mask hole as a slit, and (b) slitless, using the displacement as a measure of wavelength and therefore velocity. In Fig. 2 we show the result of comparing the slitless versus the classical measurement. Of course there is a range of velocities across NGC 7293; we are measuring its velocity field. But there is very good agreement between both measurements for each calibration point, and the average velocity of NGC 7293 turns out to be about $-30 \mathrm{~km} \mathrm{~s}^{-1}$, in good agreement with the catalogue velocity (Acker et al. 1992), which is $-28 \mathrm{~km} \mathrm{~s}^{-1}$. From this result we believe that the errors in our FOCAS slitless radial velocities are below $10 \mathrm{~km} \mathrm{~s}^{-1}$. 

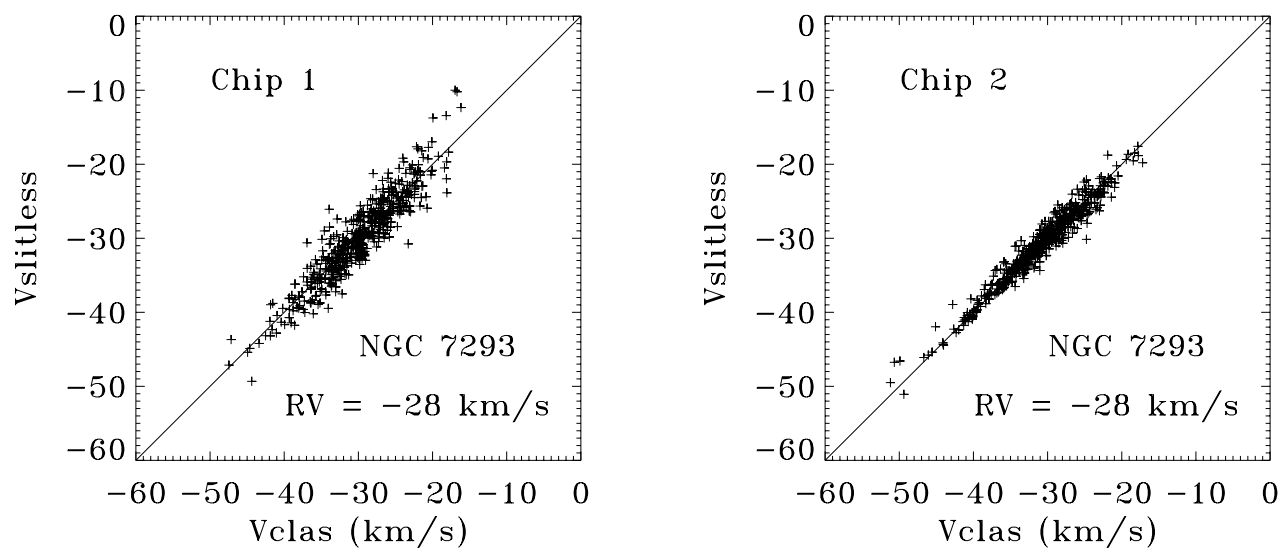

Figure 2. Slitless vs slit (classical) radial velocities across NGC 7293.
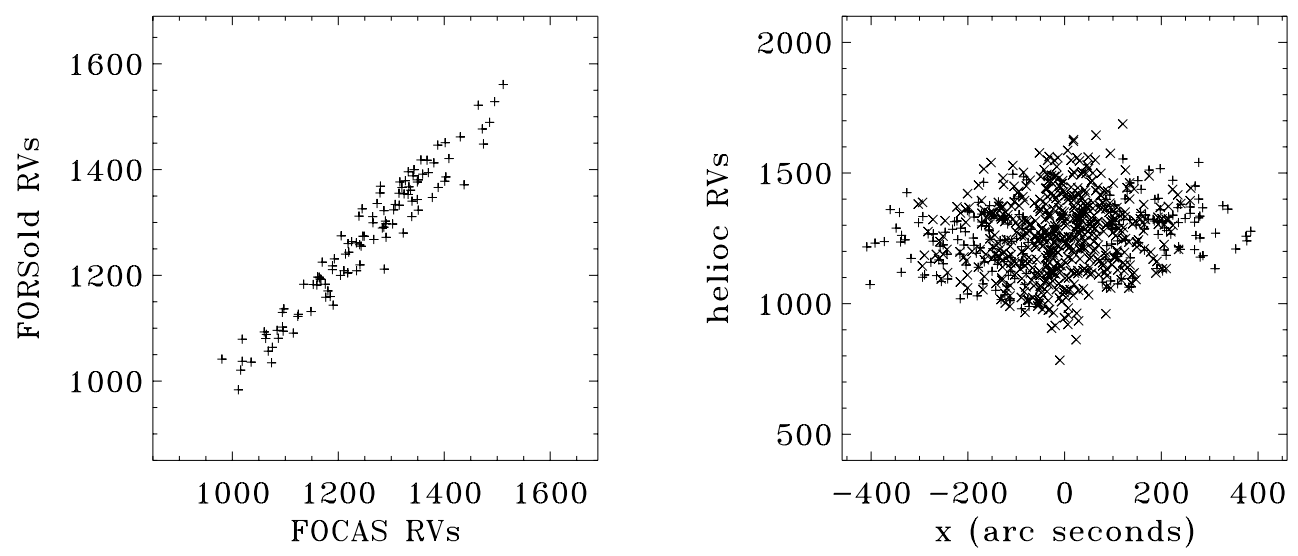

Figure 3. The left panel shows the old FORS slitless velocities vs. the FOCAS slitless velocities for 112 PNs in NGC 4697. The right panel shows a combination of old and new measurements: velocities of more than $600 \mathrm{PNs}$ as a function of their x-coordinates in arcsec relative to the center of light of NGC 4697. Crosses are FORS measurements; plus signs are FOCAS measurements. The x-axis is defined in the direction of the major axis of the galaxy, as in Méndez et al. (2001). The decrease of velocity dispersion with increasing distance to the center is obvious, even before a quantitative analysis. The effective radius of NGC 4697 is 95 arcsec. We have found several PNs as far as four effective radii from the center; the earlier study stopped at about 2.7 effective radii.

\section{New and known PNs in NGC 4697}

We have measured slitless radial velocities of 201 PNs in NGC 4697 with FOCAS. Of these PNs, 112 had been measured earlier with FORS (Méndez et al. 2001). Fig. 3a shows the FORS vs. FOCAS slitless velocities for these 112 objects. We find good agreement. Fig. $3 \mathrm{~b}$ shows the old and new measurements combined.

Among the new PNs, there are 25 located beyond three effective radii from the center of the galaxy. They allow us to extend our description of the run of the line-of-sight velocity dispersion as a function of angular distance from the center. Fig. 4 shows the 


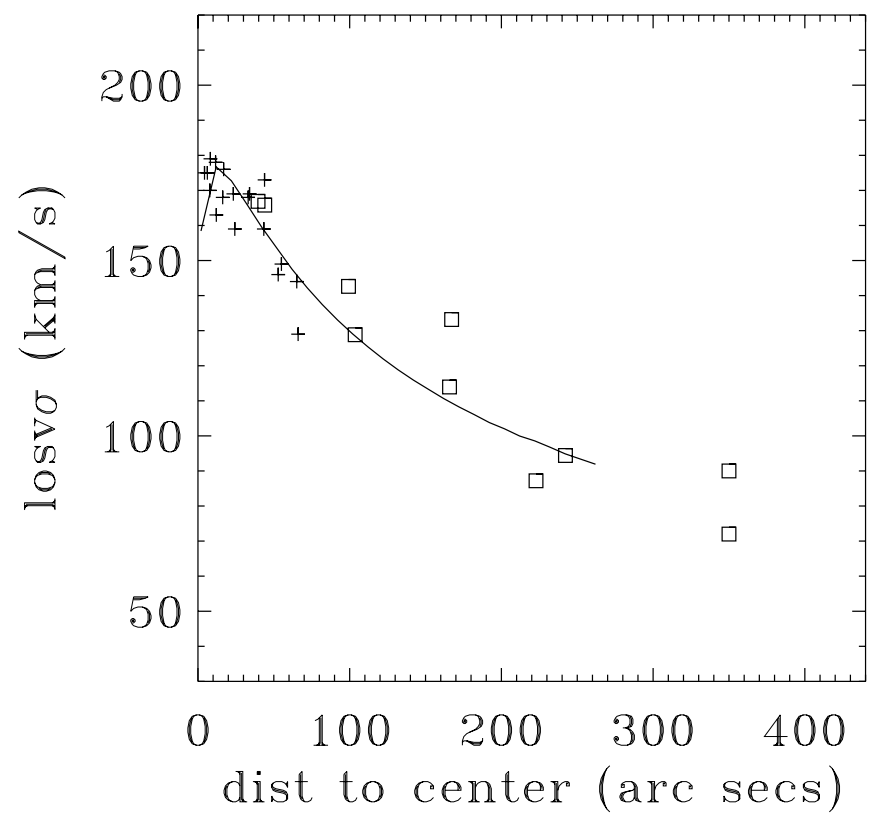

Figure 4. An update of Fig. 24 in Méndez et al. (2001). Plus signs are line-of-sight velocity dispersions $(\operatorname{losv} \sigma)$ from integrated light spectra along the major axis of NGC 4697 (Binney et al. 1990). Squares are our $\operatorname{losv} \sigma$, from the PN velocities. The FOCAS velocities gave the two new data points at an average distance of 350 arcsec. The solid line is an analytical model with a constant $M / L$ ratio (Hernquist 1990). It fits the outer points perfectly, as it did before; there is still no evidence of dark matter here.

result, which we would like to carefully express in this way: it is not that there cannot be any dark matter around NGC 4697; it is that we have failed again in our efforts to find it.

The situation (described by Romanowsky in this volume) becomes more interesting because in other cases of intermediate-mass ellipticals there is clear evidence of dark matter: NGC 5128 (Hui et al. 1995) and NGC 1344 (Teodorescu et al. 2005). As we collect more information, some degree of variety in the amount and/or distribution of dark matter appears to be emerging. We will need data from many other ellipticals before a clearer picture can be formed. The good news is that FOCAS is an excellent tool to do the job.

\section{References}

Acker, A., Ochsenbein, F., Stenholm, B., et al. 1992, Strasbourg-ESO Catalogue of Galactic Planetary Nebulae

Binney, J.J., Davies, R.L., \& Illingworth, G.D. 1990, ApJ 361, 78

Douglas, N.G., Arnaboldi, M., Freeman, K.C., et al. 2002, PASP 114, 1234

Hernquist, L. 1990, ApJ 356, 359

Hui, X., Ford, H.C., Freeman, K.C., \& Dopita, M.A. 1995, ApJ 449, 592

Méndez, R.H., Riffeser, A., Kudritzki, R.-P., et al. 2001, ApJ 563, 135

Teodorescu, A.M., Méndez, R.H., Saglia, R.P., et al. 2005, ApJ 635, 290 

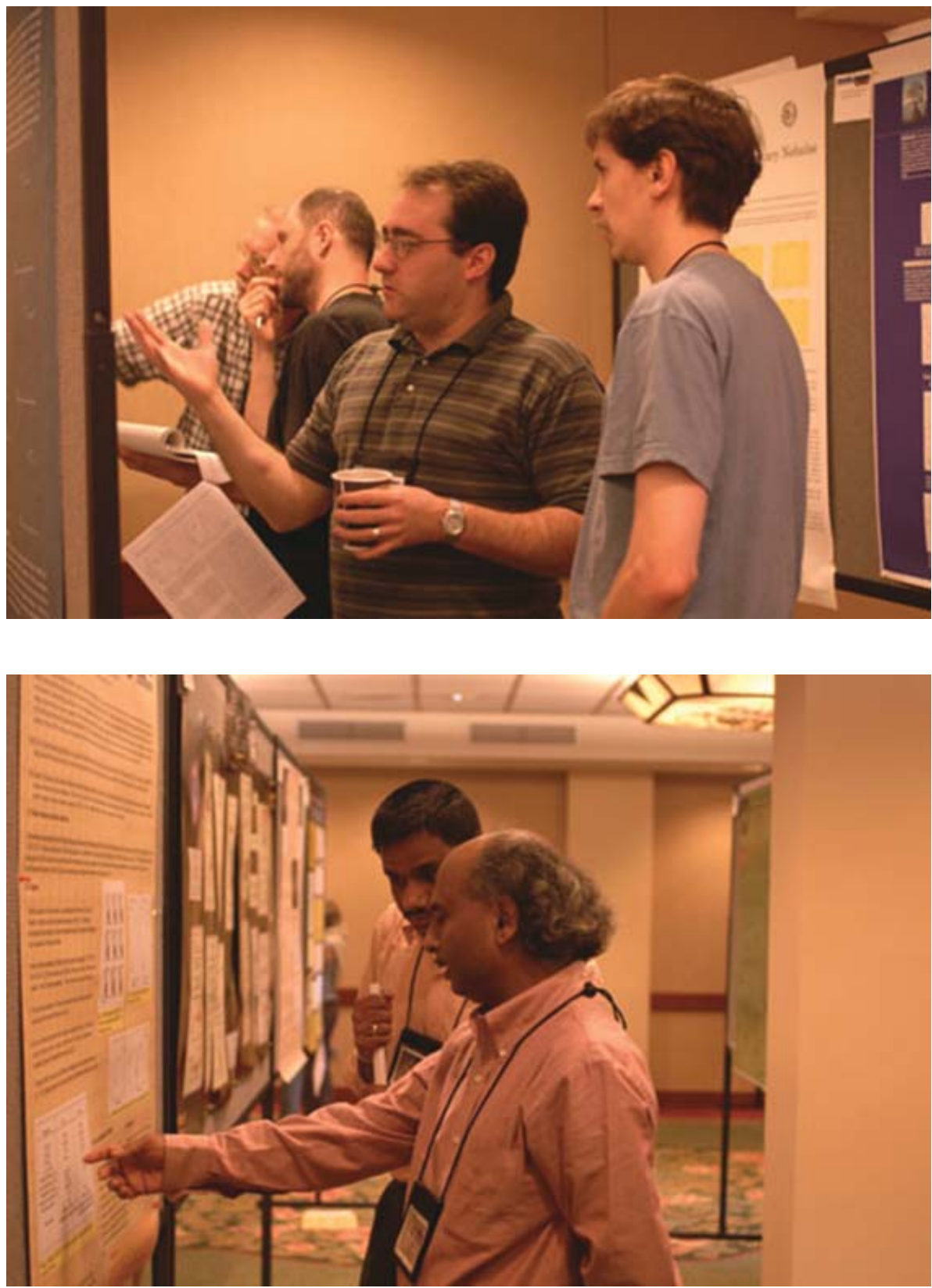

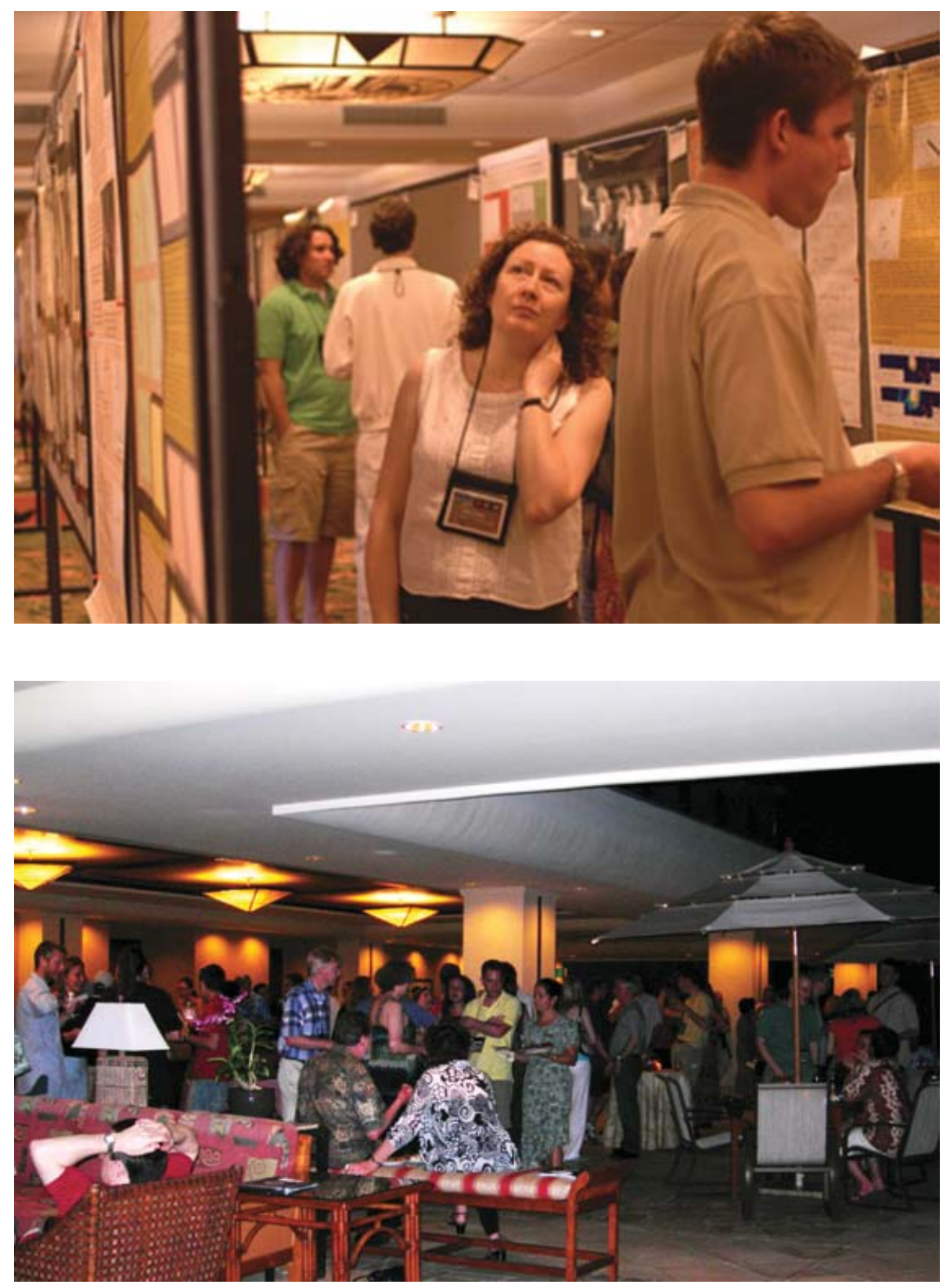